\title{
Rockburst Inducement Mechanism and Its Prediction Based on Microseismic Monitoring
}

\author{
Yongsong Li $\mathbb{D}^{1}$ and Chao Zhou $\mathbb{D}^{2}$ \\ ${ }^{1}$ School of Architecture Engineering, Huanghuai University, Zhumadian, China \\ ${ }^{2}$ Key Laboratory of Geotechnical Mechanics and Engineering of Ministry of Water Resources, Yangtze River Scientific \\ Research Institute, Wuhan, China \\ Correspondence should be addressed to Chao Zhou; zhouchao@mail.crsri.cn
}

Received 18 September 2021; Revised 21 October 2021; Accepted 25 October 2021; Published 16 November 2021

Academic Editor: Haojie Lian

Copyright (c) 2021 Yongsong Li and Chao Zhou. This is an open access article distributed under the Creative Commons Attribution License, which permits unrestricted use, distribution, and reproduction in any medium, provided the original work is properly cited.

\begin{abstract}
The rockburst disaster in the hard rock caused by excavation and unloading of deep underground caverns threatens the safety of engineering construction. In recent years, the microseismic monitoring technology, which can dynamically monitor the whole process of progressive failure of rock mass in real time, has been widely used in rockburst monitoring and early warning of underground engineering. In view of the slight rockburst in local surrounding rock during the excavation of underground powerhouse of Huanggou Pumped Storage Power Station, a rockburst microseismic monitoring system is constructed. And through the analysis of the temporal and spatial activity of microseisms during the monitoring period, the potential risk areas of rockbursts are identified and delineated. The monitoring results show that the microseismic system can effectively capture the blasting and microseismic signals during construction. The microseismic activity is closely related to the intensity of field blasting disturbance. The potential risk areas of rockburst are the upstream side arch shoulder and the intersection between lower drainage corridor and workshop installation room. The research results can provide technical support for later excavation and support of underground powerhouse caverns of Huanggou Hydropower Station.
\end{abstract}

\section{Introduction}

Rockburst is a phenomenon of rockmass dynamic instability such as fracture, spalling, ejection, and even throwing, caused by sudden release of elastic strain energy stored therein due to excavation and load removal in underground projects in high ground stress circumstances. Rockburst often occurs in hard and brittle rock masses, and they are accompanied by different degrees of cracking noises [1-4]. As underground projects go deeper, rockbursts occur more frequently. Frequent rockburst disasters during the excavation of deep underground caverns directly threaten the safety of construction personnel and equipment and delay the construction progress, especially, in severe cases. For example, on November 28, 2009, the drainage tunnel of the Jinping II Hydropower Station on the Yalong River in China's Sichuan province suffered a strong rockburst during construction, and its supporting system was seriously damaged, resulting in seven persons killed and one person injured. The rescue and cleaning work lasted for more than one month, and the direct and indirect economic losses caused by the accident were immeasurable $[4,5]$.

At present, the rockburst prediction methods applied in engineering practice at home and abroad mainly include microseismic, microgravity, electromagnetic radiation, seismology, drilling cuttings, moisture, photoelasticity, rheological method, and rebounding [6]. Among them, the microseismic method can dynamically monitor the whole process of progressive failure of rock mass in real time, effectively delineate the potential risk area of rockburst in the monitoring area, and timely evaluate and predict the stability of surrounding rock. Compared with other on-site rockburst monitoring methods, the microseismic method has obvious advantages and has been widely promoted and applied in engineering practice in recent years. 
Advanced microseismic monitoring systems have been installed in most of the deep underground projects in developed countries such as Canada, South Africa, the United States, and Australia and have been successfully applied in analyzing and determining the fracture distribution, damage state, and rockburst risk prediction [7]. In 1986, the Mentougou Coal Mine in Beijing used the 8-channel microseismic monitoring system imported from Poland to monitor the microseismic activity in the coal mining area, which was the beginning of the application of the microseismic monitoring system in China $[8,9]$. Li et al. [10] established a 16-channel microseismic monitoring system in the Fankou Lead-Zinc Mine in Guangdong province and preliminarily analyzed the microseismic activity of large blasting in deep mining area. In recent years, Feng and other scholars introduced the microseismic system into rockburst monitoring and early warning of underground caverns of such hydropower projects as Jinping, Dagangshan, and Houziyan [11-14]. Ma and other scholars applied the microseismic monitoring system to slope engineering, in order to delineate the possible rock mass fracture zone and potential slip plane of slope and provide a basis for slope engineering support [15-17]. Practices have proved that the microseismic monitoring technology has laid a good foundation for the prediction and protection of rockburst disasters in deep underground engineering.

In this paper, the microseismic monitoring system of underground powerhouse of the Huanggou Pumped Storage Hydropower Station is constructed, and based on the monitoring data of two months from the beginning, the microseismic activities induced by blasting excavation of powerhouse caverns are analyzed.

\section{General Situation of Underground Powerhouse Area of the Huanggou Hydropower Station}

The Huanggou Pumped Storage Power Station is located in Sandaohezi Town, Hailin, Mudanjiang, Heilongjiang province. Its installed capacity is $4 \times 300,000 \mathrm{~kW}$. The main structures include an upper reservoir, a water transmission system, an underground powerhouse system, a ground switch station, and a lower reservoir. The excavation size of the main and auxiliary powerhouse caverns is $163.20 \times 25.00 \times 53.80 \mathrm{~m}$ $(L \times W \times H)$, and the top arch elevation is $178.60 \mathrm{~m}$. The excavation size of the main transformer room is $127.10 \times 21.20$ $\times 22.40 \mathrm{~m}(L \times W \times H)$, and the top arch elevation is $175.10 \mathrm{~m}$. The excavation size of the tail gate chamber is $94.90 \times 11.40 \times 20.30 \mathrm{~m}(L \times W \times H)$, and the top arch elevation is $159.20 \mathrm{~m}$. The axial direction of the powerhouse is $\mathrm{N} 49^{\circ} \mathrm{W}$, and the main transformer chamber and tailgate chamber are arranged parallel to the powerhouse. The center distance between the main powerhouse and main transformer chamber is $61.30 \mathrm{~m}$, and that between the main transformer chamber and tailgate chamber is $44.35 \mathrm{~m}$.

The area occupied by the power station is topographically a small tectonic basin in the low and medium-height mountains and river valleys formed by tectonic erosion.
The underground powerhouse area has a hilly terrain which has been mildly cut, with the elevation of hills between 470 and $530 \mathrm{~m}$ and the ground surface slope between $20^{\circ}$ and $40^{\circ}$. The underground powerhouse is $350 \sim 400 \mathrm{~m}$ in burial depth, and its surrounding rock is mainly hard and complete alaskite. The geological structure of such surrounding rock is not well developed, and two small faults, $\mathrm{f}_{33}$ and $\mathrm{f}_{34}$, with steep dip angles, $3-5 \mathrm{~mm}$ in width and modest extension, are found therein. The main transformer chamber passes through two steeply inclined faults, $f_{31}$ and $f_{32}$. The $f_{31}$ fault is $0.3 \sim 1.10 \mathrm{~m}$ wide, and the latter is $0.05 \mathrm{~m}$ wide. Their fillings are mainly cataclastic rock and rock fragments. In this area, there are three groups of steep-dip joints (NWW, NE, and NEE, respectively) and a group of NEE-direction gentle-dip joints in the rock mass. The joint spacing is generally $0.5 \sim 2.5 \mathrm{~m}$, and most of them are in a closed state. The surrounding rocks of the powerhouse are mostly type II surrounding rocks, and their integrity is relatively complete to complete (see Figure 1). According to the measurement of the in situ stress and the analysis of the regression inversion results of the stress field, the maximum principal stress in the underground powerhouse area is 11.0 15.0 MPa, which is a medium stress.

In the initial stage of excavation of the underground powerhouse of the hydropower station, the surrounding rocks of various locations have experienced different degrees of slab and rockburst (see Figure 2). In order to understand the stability of the surrounding rock in the subsequent excavation process, the microseismic monitoring system is introduced to monitor the underground powerhouse in real time, so as to identify and delineate the potential instability hazard areas that affect the stability of the surrounding rock and provide guidance for safe construction.

\section{Microseismic Monitoring System in Underground Powerhouse Area}

3.1. Principles of Microseismic Monitoring and Positioning. When the rock mass is disturbed by external forces, microcracks will occur in the rock mass, which is usually accompanied by elastic waves, releasing strain energy and generating microseismic events. If a certain number of sensors are arranged in the rock mass around the seismic source to form a spatial array, when a microseismic event occurs in the monitoring body, the elastic wave generated by it will propagate outward through the surrounding medium and be received by sensors installed in the hole close to the rock wall. The sensor converts microseismic signals into electrical signals and sends them to the signal acquisition instrument through cables. The signal acquisition instrument converts the electrical signals into digital signals and transmits them to the analysis computer. By analyzing and processing microseismic events with waveform processing software and demonstrating them with $3 \mathrm{D}$ visualization software, the time, space position, and intensity information of rock microseismic events can be obtained, that is, the three elements of "time, space, and intensity" of rock microfracture [18]. Figure 3 is a schematic diagram of the principle of microseismic monitoring. 


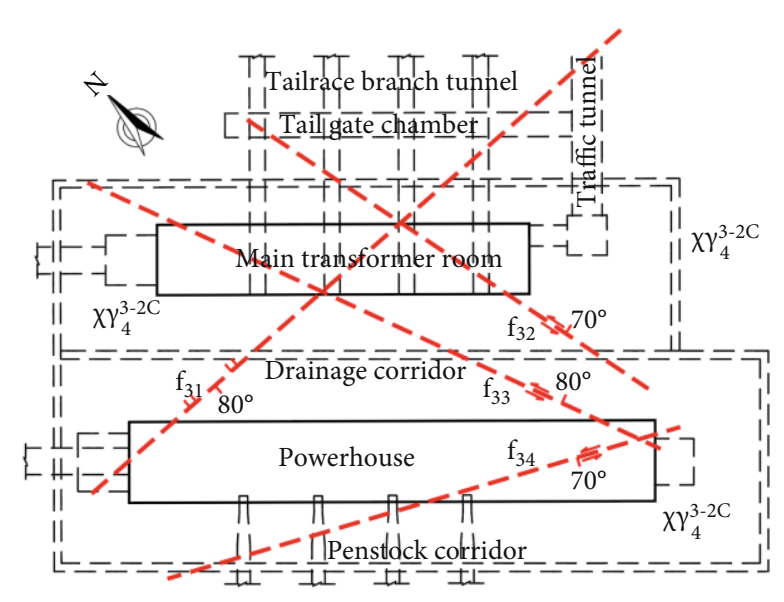

FIGURE 1: Planar graph of underground powerhouse area at elevation $172.60 \mathrm{~m}$.

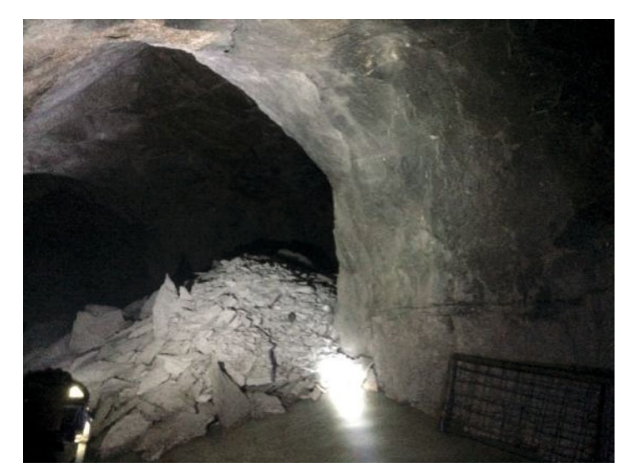

Figure 2: Rockburst at the intersection between lower drainage corridor and tailrace branch tunnel no. 1 .

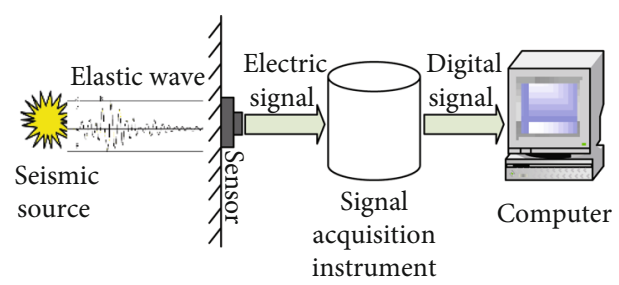

FIGURE 3: Schematic diagram of the principle of microseismic monitoring.

Microseismic source positioning is a method to determine the location of the rock fracture source according to the elastic wave propagation speed in the rock medium by monitoring the arrival of the elastic wave generated by the rockmass fracture. The principle of source location is shown in Figure 4. Microseismic positioning methods can be divided into regional positioning method and point positioning method (time difference positioning method). At present, several commonly used positioning methods mainly include the least square method, Bayesian positioning method, relative positioning method (ATD), slowness dispersion method, simplex positioning method, and Geiger positioning method [19-22]. The Geiger positioning method is an application of the Gauss-Newton method. It is suitable

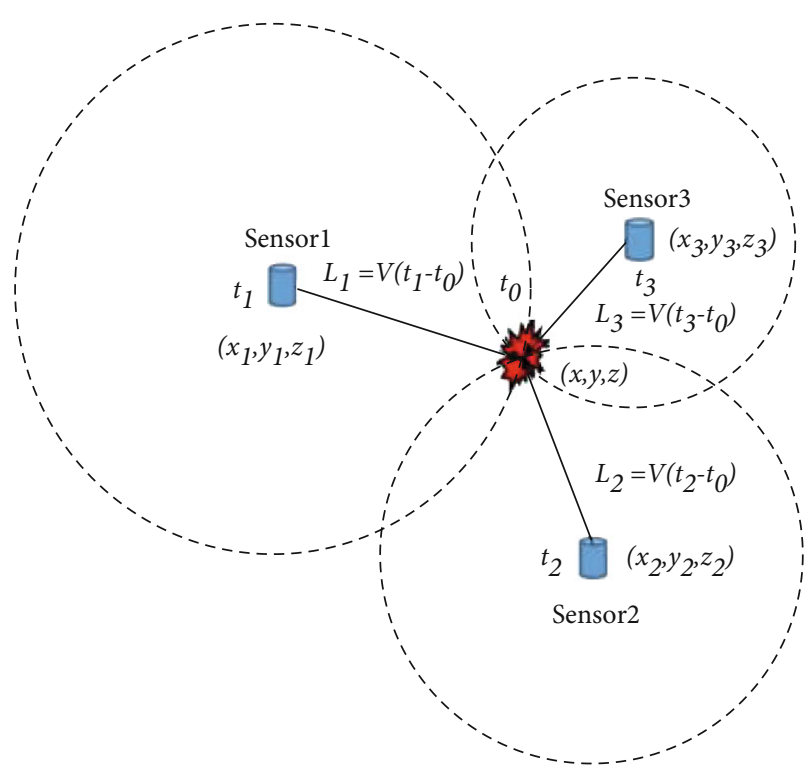

Figure 4: Schematic diagram of the source location principle.

for small-area seismic events with high positioning accuracy, and this method requires relatively loose positioning conditions for events, and there are more reasonable positioning events. The Canadian ESG microseismic monitoring system used in this article uses the Geiger positioning method based on the time difference positioning principle to locate the source event. In the sensor array arranged around the microseismic source monitoring area, the relative distance between the sensors is known. The difference in the arrival time of the elastic wave picked up by each sensor in the array is called the time difference. The time difference provides the distance information between the source event and the sensor array, and the location of the source event can be determined by selecting a suitable seismic wave velocity model.

Geiger positioning method is to approach the final result through iteration from a given test point $O(x, y, z, t)$. In each iteration, a correction vector $\Delta \theta(\Delta x, \Delta y, \Delta z, \Delta t)$ is calculated based on the least square method, and the vector $\Delta \theta$ is added to the result of the previous iteration (test point) to obtain a new test point and then judge this whether the new test point meets the requirements, if it meets the requirements. If it meets the requirements, the coordinates of this point will be the source position. If not, continue iteration. The result of each iteration is generated by the following time distance equation (1) (where $x, y, z$, and $t$ are known numbers generated by artificial setting or iteration).

$$
\left(x_{i}-x\right)^{2}+\left(y_{i}-y\right)^{2}+\left(z_{i}-z\right)^{2}=v_{p}^{2}\left(t_{i}-t\right)^{2},
$$

where $(x, y, z)$ are the coordinates of the test point, the initial value of which is set manually; $t$ is the time when the event occurs, and its initial value is set manually; $\left(x_{i}, y_{i}, z_{i}\right)$ is the spatial position of the $i$-th sensor coordinates; $t_{i}$ is the time of the $P$ wave signal received by the $i$-th sensor; $v_{p}$ is the propagation speed of the $P$ wave in the rock mass. 
For the arrival time $t_{o, i}$ of the $P$ wave received by the $i$-th sensor, the first-order Taylor expansion of the arrival time calculated by the test point coordinates can be expressed as

$$
t_{o, i}=t_{c, i}+\frac{\partial t_{i}}{\partial x} \Delta x+\frac{\partial t_{i}}{\partial y} \Delta y+\frac{\partial t_{i}}{\partial z} \Delta z+\frac{\partial t_{i}}{\partial t} \Delta t
$$

where $t_{c, i}$ is the time for the $P$ wave calculated from the test point coordinates to reach the $i$-th sensor. In equation (2),

$$
\begin{aligned}
\frac{\partial t_{i}}{\partial x} & =\frac{x_{i}-x}{v_{p} R}, \\
\frac{\partial t_{i}}{\partial y} & =\frac{y_{i}-y}{v_{p} R}, \\
\frac{\partial t_{i}}{\partial z} & =\frac{z_{i}-z}{v_{p} R}, \\
\frac{\partial t_{i}}{\partial t} & =1, \\
R & =\sqrt{\left(x_{i}-x\right)^{2}+\left(y_{i}-y\right)^{2}+\left(z_{i}-z\right)^{2}} .
\end{aligned}
$$

For $N$ sensors, $N$ equations can be obtained and written in the form of matrix.

$$
A \Delta \theta=B,
$$

where

$$
\begin{aligned}
A & =\left[\begin{array}{cccc}
\frac{\partial t_{1}}{\partial x} & \frac{\partial t_{1}}{\partial y} & \frac{\partial t_{1}}{\partial z} & 1 \\
\frac{\partial t_{2}}{\partial x} & \frac{\partial t_{2}}{\partial y} & \frac{\partial t_{2}}{\partial z} & 1 \\
\vdots & \vdots & \vdots & \vdots \\
\frac{\partial t_{n}}{\partial x_{1}} & \frac{\partial t_{n}}{\partial v} & \frac{\partial t_{n}}{\partial z} & 1
\end{array}\right] \\
\Delta \theta & =\left[\begin{array}{c}
\Delta x \\
\Delta y \\
\Delta z \\
\Delta t
\end{array}\right] \\
B= & {\left[\begin{array}{c}
t_{o 1}-t_{c 1} \\
t_{o 2}-t_{c 2} \\
\vdots \\
t_{o n}-t_{c n}
\end{array}\right] }
\end{aligned}
$$

The modified vector is obtained by solving equation (3) with Gaussian water elimination method.

$$
A^{T} A \Delta \theta=A^{T} B
$$

$$
\Delta \theta=\left(A^{T} A\right)^{-1} A^{T} B .
$$

After the correction vector is obtained from equation (5), the iteration is continued with $(\theta+\Delta \theta)$ as the new test point until the error requirement is met.

3.2. Establishment of Microseismic Monitoring System. A high-precision microseismic monitoring system of Canada's ESG (Engineering Seismology Group) is adopted in the project, which includes acceleration sensors, cables, Paladin signal collection system, and HANS data processing system. The underground powerhouse area in the project is formed by excavation by 7 layers, with the thicknesses of $10 \mathrm{~m}$, $8 \mathrm{~m}, 8.1 \mathrm{~m}, 6.6 \mathrm{~m}, 7.4 \mathrm{~m}, 7.5 \mathrm{~m}$, and $6.2 \mathrm{~m}$ from top to bottom. When the microseismic system is being set up, the excavation of the third layer has finished, the tailgate chamber and busbar tunnel have reached their middle point of excavation, and the main transformer chamber, upper/middle/lower drainage corridors, ventilation tunnel, and traffic tunnel have all been excavated to full capacity. According to the excavation status of the underground powerhouse area, 18 sensors are arranged in the excavated upper/middle drainage corridors to form a spatial array, focusing on monitoring and analyzing the influence of further excavation disturbance on the stability of the surrounding rock of the powerhouse roof arch and upstream side wall. Figure 5 is a schematic diagram of the layered excavation of the underground powerhouse and the location of the sensors.

Figure 6 is a schematic diagram of the spatial arrangement of sensors. Six and eight uniaxial sensors are, respectively, arranged in the upper and middle drainage corridors of the underground powerhouse system, three uniaxial sensors are arranged in the main transformer room, one uniaxial sensor is arranged in the traffic tunnel between the powerhouse and the main transformer room, and the monitoring center is placed in the traffic tunnel. The sensor mounting hole has a diameter of $50 \mathrm{~mm}$ and a depth of about $4 \mathrm{~m}$. When being installed, the front end of the sensor is fixed at the bottom of the hole with anchor resin. The sensor is in point contact with the rock mass and can receive elastic wave signals from all directions. After the microseismic system is in position, the operation of the sensor was verified by a manual percussion test.

3.3. Selection of Velocity Model. The effective selection of the velocity model is very important to the positioning accuracy of the source event and thus the positioning error. After the installation of the system, a total of 10 manual knocking experiments are carried out in the upper and middle drainage corridors to decide the speed model. According to the available information, the average wave velocity in the rock mass in the underground powerhouse area is $4700 \sim 5100 \mathrm{~ms}^{-1}$. The system wave speed adopts 17 different wave speed values between 4700 and $5100 \mathrm{~ms}^{-1}$. With reference to the principle of event location and capture and the method of waveform processing discussed in reference [19], ten experimental points of manual percussion are systematically located, and the average error of location results is obtained. The correlation analysis of wave velocity and event location error is 


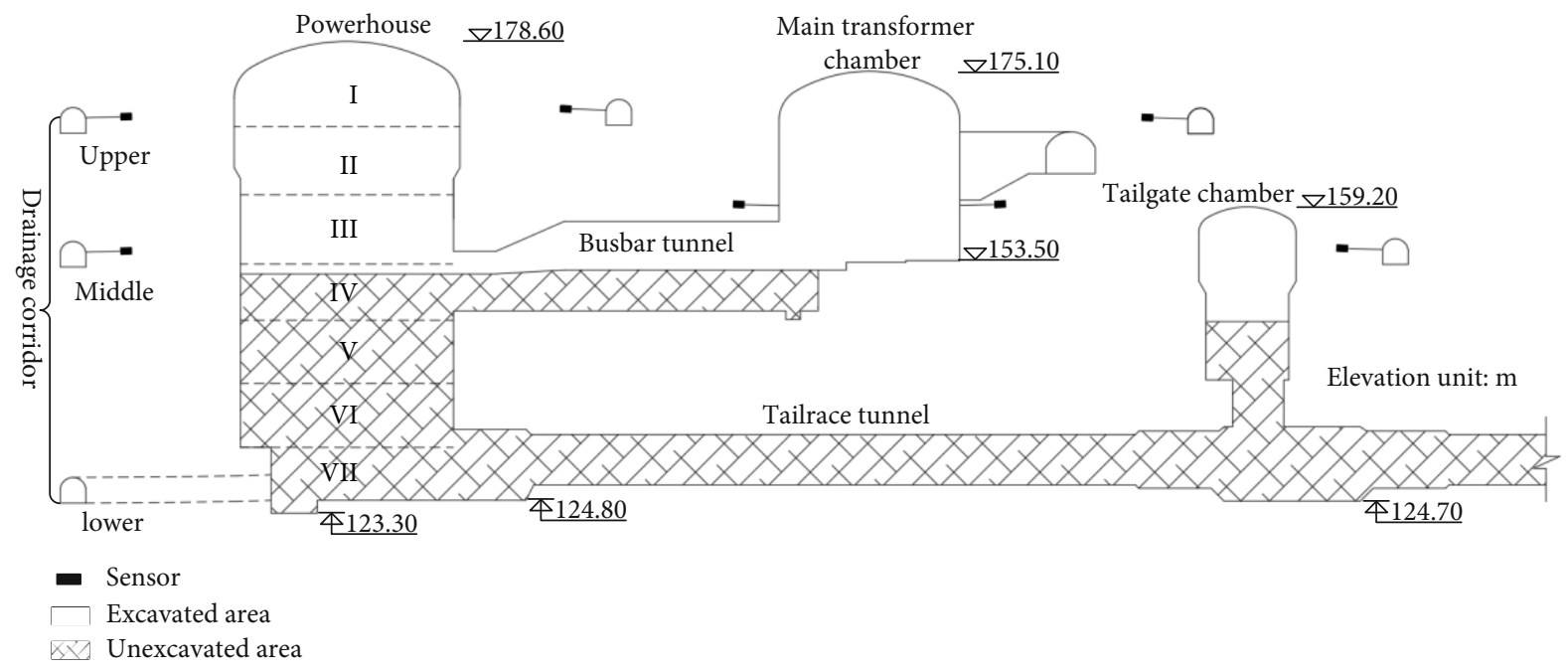

FIGURE 5: Schematic diagram of layered excavation of underground powerhouse and sensor positions.

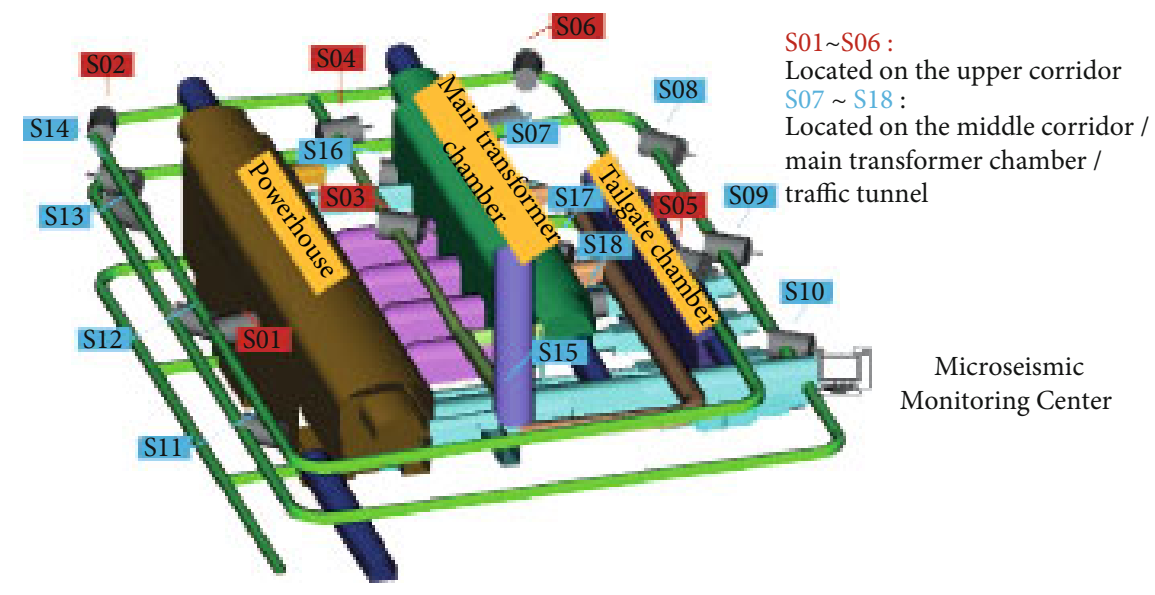

FIGURE 6: Schematic diagram of sensor layout in underground powerhouse area.

carried out, and the results are shown in Figure 7. Finally, the optimal $P$ wave velocity of rock mass in the monitoring area is $5150 \mathrm{~ms}^{-1}$, at which the minimum positioning error is $5.4 \mathrm{~m}$, and the positioning accuracy of the system meets the requirements of monitoring [11].

\section{Analysis of Microseismic Monitoring Results}

Between September 30, 2017, and November 30, 2017, the microseismic monitoring system has captured 123 microseismic events, two occurrences per day on average, and 54 blasting events, roughly one per day on average. Figure 8 shows the typical microseismic and blasting event signal wave monitored by the system. The microseismic signal has unique wave component and short duration, its amplitude is mostly concentrated between tens and hundreds of millivolts, and its frequency is mainly distributed in the middle- and low-frequency bands below $500 \mathrm{~Hz}$. The blasting wave has multiple waveforms superimposed on each other in a window, with a long duration, large amplitude, and high frequency.

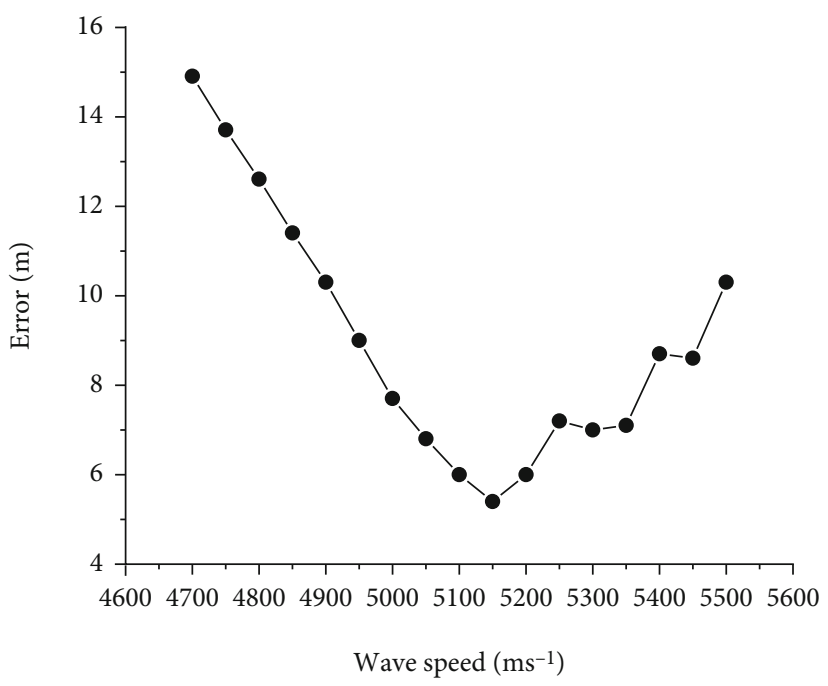

FIGURE 7: Relation curve between wave velocity and position error. 


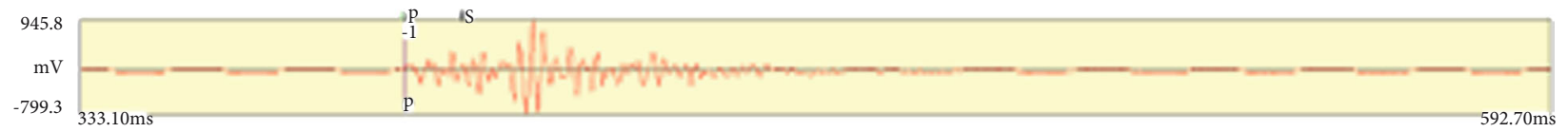

(a) Microseismic signal

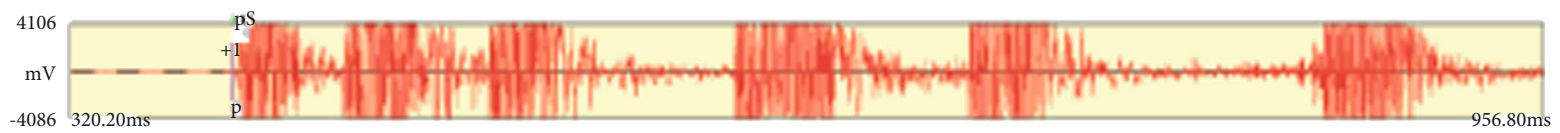

(b) Blasting signal

Figure 8: Typical microseismic and blasting event signal waveforms.

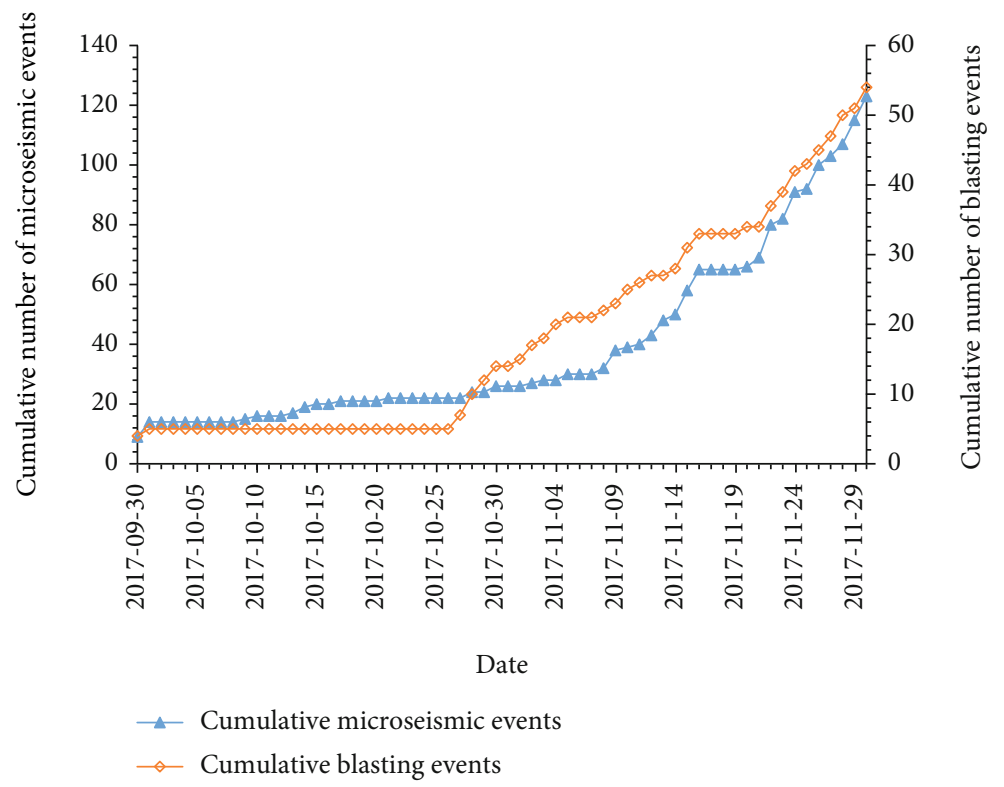

FIGURE 9: Evolution of accumulative microseismic events and blasting events over time.

4.1. Temporal Distribution of Microseismic Events. By analyzing the temporal distribution of microseismic events, it is possible to understand the temporal characteristics of the rock microcrack's initiation, development, and aggregation and find out the relationship between rock microcracks and construction disturbances such as underground cavern excavation [12]. Figure 9 shows the evolution of accumulated microseismic events and blasting events in the monitoring area of the underground powerhouse from September 30 to November 30, 2017. It can be seen from the figure that from September 30 to October 26, the evolution curve of cumulative microseismic events with time is gentle and the increment range is small, which indicates that there are few microfracture events in rock mass and the rockburst risk is low. There is no increase in cumulative blasting events. During this period of time, the construction was halted for some reason. The blasting operation was completely off at the site, the rock mass was almost not affected by the disturbance of external force, and the microseismic activity was not obvious After the construction was resumed, the cumulative microseismic events and blasting events showed an obvious increasing trend with time, and there was a positive correlation between the two types of events. During this period, the microfracture events in rock mass were relatively active, and the rockburst risk was relatively high, which was obviously affected by the on-site excavation and blasting. It can be seen that external forces such as construction disturbance are the main factors inducing microseismic events.

4.2. Spatial Distribution of Microseismic Events. By means of the spatial distribution of microseismic events, the microfracture area of rock mass can be effectively identified, and then, the potential rockburst risk area of the underground powerhouse area can be predicted. The concentration area of microseismic events can be divided by analyzing the density and frequency of the spatial distribution of microseismic events. Figure 10 shows the spatial distribution of microseismic events in the monitoring area from September 30 to November 30, 2017. In this figure, a sphere represents a microseismic event, and its size represents energy, with a bigger sphere standing for greater energy and different colors representing different moment magnitudes. The microseismic events are mainly concentrated in the arch shoulder on the upstream side of the powerhouse with an elevation ranging from $150 \mathrm{~m}$ to $180 \mathrm{~m}$ (recorded as zone 


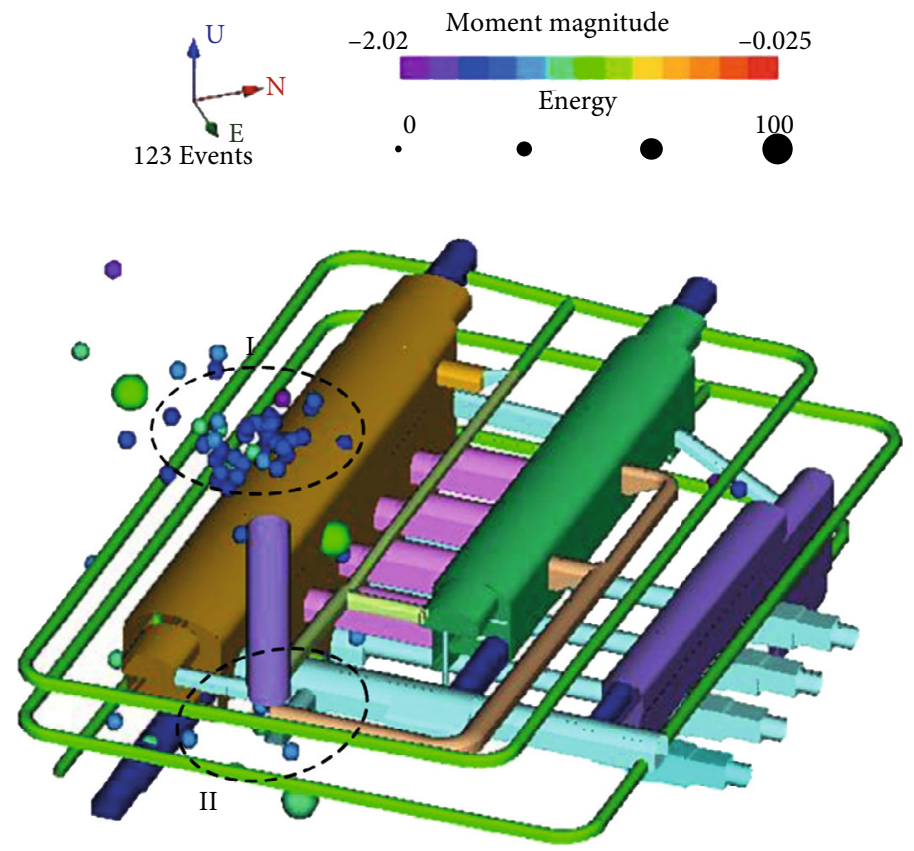

(a) Axonometric view

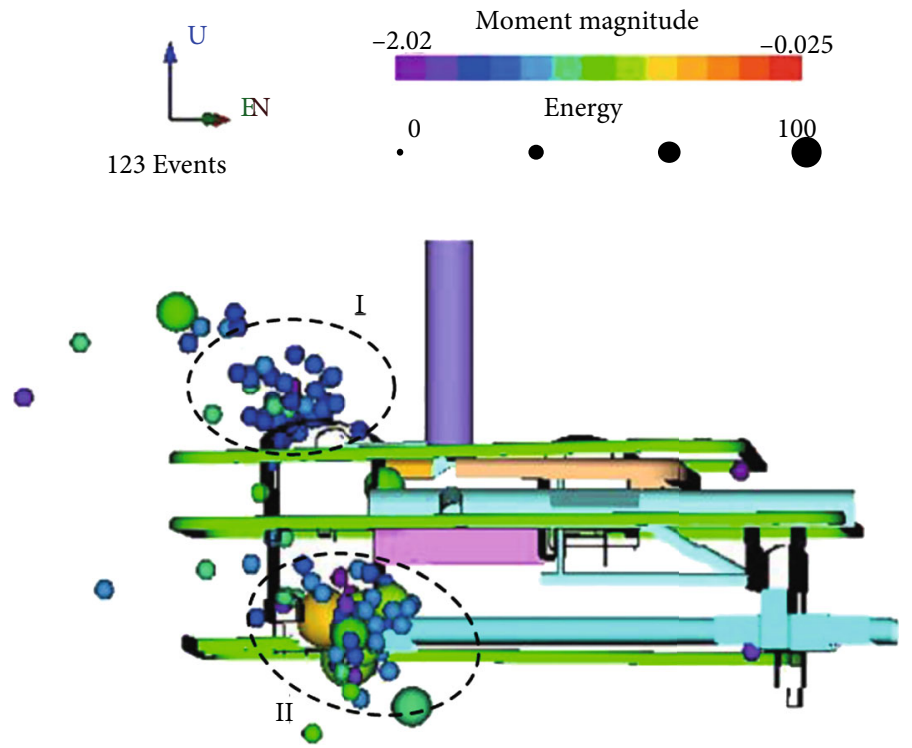

(b) Front view

FIGURE 10: Spatial distribution of microseismic events.

I) and the intersection of the lower drainage corridor and the powerhouse installation room with an elevation ranging from $110 \mathrm{~m}$ to $140 \mathrm{~m}$ (recorded as zone II). Combined with the on-site construction of underground powerhouse and on-site survey analysis, microseismic events in these two zones are distributed along faults $\mathrm{f}_{31}$ and $\mathrm{f}_{34}$. Microseismic events concentrate in the above zones because of dynamic disturbances such as mechanical vibration and blasting vibration during the excavation of the powerhouse. However, there is a main fault developed in the microseismic accumulation area of the surrounding rock, and the stress is likely to concentrate on the structural surface of the fault with lower strength, which induces microcracking of the rock.
4.3. Preliminary Analysis of Microseismic Events. After processing the microseismic data in two months and analyzing the construction of the underground powerhouse caverns, the concentration area of microseismic events is mainly caused by the dynamic disturbances brought about by the vibration of construction machineries and blasting operations. The magnitude, energy, and focal radius of microseismic events in zone I are small, while those in zone II are larger. At present, there is no large-scale rockburst in the monitoring area, but rock mass collapses occur occasionally in some parts of the microseismic event concentration area (see Figure 11). Microcracks constantly occur in rock mass, indicating the potentially dangerous places for rockburst, 


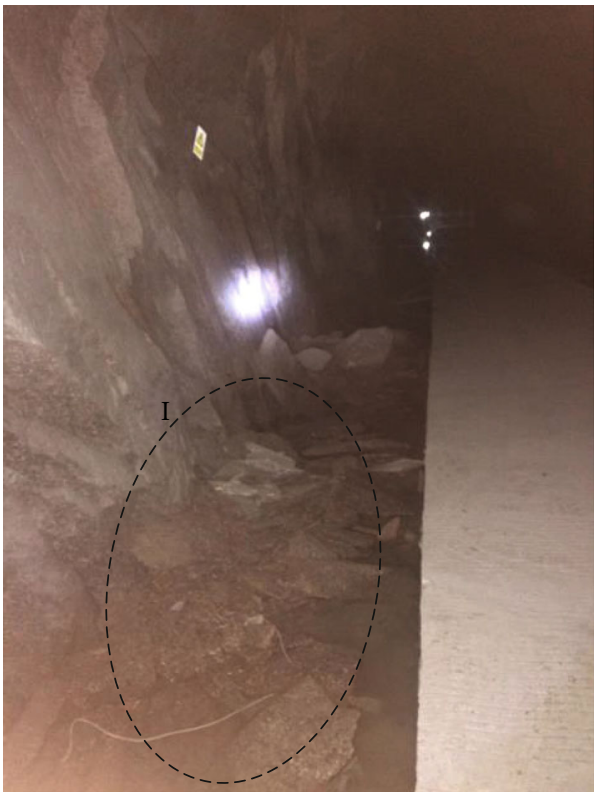

(a) Located near the left $0+40$ of the pile number factory on the inner side wall of the upstream side of the middle drainage corridor (elevation $155 \mathrm{~m}$ )

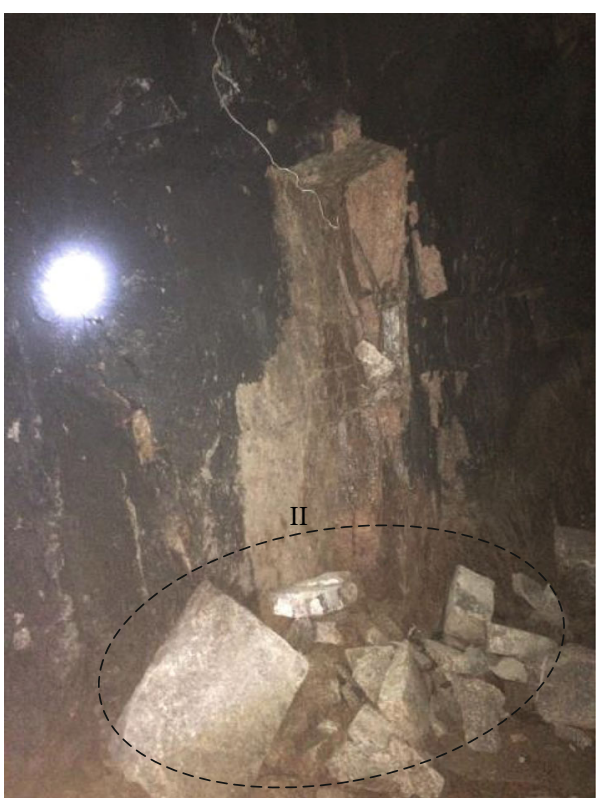

(b) Located near the left $0+35$ of the pile number factory on the inner side wall (elevation $125 \mathrm{~m}$ ) on the upstream side of the lower drainage corridor

FIgURE 11: Collapses of rock mass in microseismic accumulation area.

making it necessary to pay attention to microseismic activities in such places. All the microseismic events have been accurately captured by the monitoring system, which proves the reliability of this microseismic system in rockburst prediction and early warning as well as the evaluation of the stability of surrounding rocks.

The prevention of rockburst during the construction phase mainly includes optimizing the stress of the surrounding rock, optimizing the physical and mechanical characteristics of the surrounding rock, and comprehensively strengthening the surrounding rock. The existing microseismic monitoring results show that the rockburst risk in the underground powerhouse area is mainly a time-delay rockburst. The engineering prevention and control of rockburst mainly include the following. (1) During blasting, smooth blasting should be enhanced, and the excavation section should be smoothly treated to reduce stress concentration and effectively improve the quality of the excavation. (2) Cold water is often sprayed on the excavation surface or high-pressure water injection is drilled to improve the physical and mechanical properties of the surrounding rock. (3) Shorten the excavation footage, which generally does not exceed $2 \mathrm{~m}$. After excavation, the initial anchor net shotcrete support will be used to reduce the exposure time and area of the rock mass. (4) Set up reasonable surrounding rock support and reinforcement measures. For example, strengthen the support of the surrounding rock in zone 1 and zone 2 and increase the length of bolts.

\section{Conclusion}

This paper takes the underground powerhouse area of the Huanggou Pumped Storage Hydropower Station as an example and uses a microseismic monitoring system to monitor and forecast rockbursts. Practices have proved that the microseismic system can effectively capture highfrequency blasting and low-frequency microseismic events. The monitoring results in two months show that the frequency of microseismic events in the underground powerhouse area is positively correlated with the construction frequency of blasting excavation. The microseismic events are mainly concentrated at the intersection of the arch shoulder on the upstream side of the powerhouse as well as at intersection between the lower drainage corridor and the powerhouse installation room and distributed in strips along the faults. At present, there are no large-scale rockbursts in the monitoring area, but close attention should be paid to the influence of construction intensity on the stability of surrounding rocks in the microseismic concentration area. Finally, targeted engineering prevention and control measures for rockbursts are proposed. This lays a foundation for the evaluation of the powerhouse's stability.

\section{Data Availability}

The data used to support the findings of this study are included within the article.

\section{Conflicts of Interest}

The authors declare no conflict of interest.

\section{Acknowledgments}

This work is partially supported by key scientific research projects of universities and key scientific and technological 
projects in Henan Province, which numbers are 21A440015, 22A570007, and 212102310601, respectively.

\section{References}

[1] M. F. Cai, D. Ji, and Q. F. Guo, "Study of rockburst prediction based on in-situ stress measurement and theory of tnergy accumulation caused by mining disturBance," Chinese Journal of Rock Mechanics and Engineering, vol. 32, no. 10, pp. 19731980, 2013.

[2] M. He, H. Xie, S. Peng, and Y. Jiang, "Study on rock mechanics in deep mining engineering," Chinese Journal of Rock Mechanics and Engineering, vol. 24, no. 16, pp. 2803-2813, 2005.

[3] G. S. Su, J. Q. Jiang, X. T. Feng, and C. Mo, "Experimental study of ejection process in rockburst," Chinese Journal of Rock Mechanics and Engineering, vol. 35, no. 10, pp. 1990-1999, 2016.

[4] Q. Yu, Study on Rockburst Nucleation Process and Early Warning Method of Deep-Buried Tunnels, The doctoral dissertation of Dalian University of Technology, 2016.

[5] C. S. ZHANG, N. LIU, and W. J. CHU, "Inducement mechanism and case analysis of tectonic rockburst in jinping II deep buried tunnel," Chinese Journal of Rock Mechanics and Engineering., vol. 34, no. 11, pp. 2242-2250, 2015.

[6] F. Z. Jiang, X. D. Xiang, and D. S. Zhu, “The current status and developing tendency of rock blasting forecast at home and abroad," Industrial Safety and Environmental Protection, vol. 8, pp. 19-22, 2003.

[7] A. J. Mendecki, Seismic Monitoring in Mines, Chapman and Hall Press, London, 1996.

[8] Z. H. Lu and Z. Liancheng, "Evaluation of near-field monitoring efficiency of tremers in Mentougou mine," Earthquake, vol. 5, pp. 32-39, 1989.

[9] C. X. Yang, Z. Q. Luo, G. B. Hu, and X. M. Liu, "Application of a microseismic monitoring system in deep mining," Journal of University of Science and Technology Beijing, vol. 14, no. 1, pp. 6-8, 2007.

[10] S. L. Li, X. G. Yin, and W. D. ZHENG, “Cezar Trifu. Research of multi-channel microseismic monitoring system and its application to Fankou lead-zinc mine," Chinese Journal of Rock Mechanics and Engineering, vol. 24, no. 12, pp. 20482053, 2005.

[11] X. T. Feng, B. R. Chen, C. Q. Zhang, S. J. Li, and S. Y. Wu, Mechanism, Warning and Dynamic Control of Rockburst Development Processes, Science Press, Beijing, 2013.

[12] B. H. Zhang, J. H. Deng, and M. Z. Gao, "Safety evaluation research based on microseismic monitoring in underground powerhouse of hydropower station," Chinese Journal of Rock Mechanics and Engineering, vol. 31, no. 5, pp. 937-944, 2012.

[13] B. Li, F. Dai, N. W. Xu, Y. G. Zhu, C. X. I. A. O. Sha, and G. He, "Microseismic monitoring system and its engineering applications of deep-buried underground powerhouse," Chinese Journal of Rock Mechanics and Engineering, vol. 33, no. S1, pp. 3375-3383, 2014.

[14] B. H. Jie, Z. N. Zhao, B. R. Chen, Y. X. Xiao, and G. L. Feng, "Regularity of spatio-temporal distribution of rockburst in deep-buried long tunnels based on microseismic monitoring signals," Journal of Yangtze River Scientific Research Institute, vol. 29, no. 9, pp. 69-73, 2012.

[15] K. Ma, C. A. Tang, L. C. Li et al., "Reinforcement effects of anti-shear gallery of Dagangshan right bank slope based on microseismic monitoring and numerical simulations," Chinese Journal of Rock Mechanics and Engineering, vol. 32, no. 6, pp. 1239-1247, 2013.

[16] N. W. Xu, C. A. Tang, C. Sha, Z. Z. Liang, J. Y. Yang, and Y. Y. Zou, "Microseismic monitoring system establishment and its engineering applications to left bank slope of jinping I hydropower station," Chinese Journal of Rock Mechanics and Engineering, vol. 29, no. 5, pp. 915-925, 2010.

[17] N. W. Xu, C. A. Tang, Z. Zhou, C. Sha, and Z. Z. Liang, "Stability analysis of hydraulic rock slope based on threedimensional numerical simulation and microseismic monitoring," Chinese Journal of Rock Mechanics and Engineering, vol. 29, no. 7, pp. 1373-1381, 2013.

[18] G. Z. Cai and C. Z. Yu, "Schedule and excavation of underground powerhouse for hydropower station," Water Resources \& Hydropower of Northeast, vol. 34, no. 11, pp. 1-2, 2016.

[19] S. D. Bing and Y. S. Pan, "Microseismic orientation method for mine and its application," Coal Mining Technology, vol. 12, no. 5, pp. 1-4, 2007.

[20] Y. X. Xiao, X. T. Feng, J. A. Hudson, B. R. Chen, G. L. Feng, and J. P. Liu, "ISRM suggested method for in situ microseismic monitoring of the fracturing process in rock masses," Rock Mechanics and Rock Engineering, vol. 49, no. 1, pp. 343-369, 2016.

[21] H. Y. Wang, "Study of precise localization of microseismic events in a rock mass," Engineering Blasting, vol. 7, no. 3, pp. 5-8, 2001.

[22] L. L. Chen, H. Lian, Z. Liu, H. B. Chen, E. Atroshchenko, and S. P. A. Bordas, "Structural shape optimization of three dimensional acoustic problems with isogeometric boundary element methods," Computer Methods in Applied Mechanics and Engineering, vol. 355, pp. 926-951, 2019. 